\title{
Design of Remote Fault Monitoring System Based on WAMP Press
}

\author{
Yuying Shao, Zhengquan Lv \\ Shanghai municipal electric power company \\ Shanghai, China
}

\begin{abstract}
For realizing the network and information of highend printing machines, the remote fault monitoring system based on Apache, MySQL and PHP under the Windows system (WAMP) for network environment is presented. The open source environment of WAMP is used in this system, which can easily set up a remote site and realize the network system of the $b / s$ structure, and is convenient for secondary development. Designing a data transfer interface based on TCP/IP protocol that allows the clients to transfer the field device data to the site server and store in the local database, which is also used to display the current status, publish historical data and diagnose the remote troubleshooting. The system is current, and the database structure can be changed depending on the object. The actually run showed that the system was running stable and normal data transfer.
\end{abstract}

Keywords-remote monitoring; WAMP; TCP/IP protocol; printing presses

\section{INTRODUCTION}

With the increasing improvement of people's living standards in China, and the demands for newspapers and advertising are also increasing, which has a higher requirement for the rotary printing machine, such as the high speed and high quality prints, quickly preparing, short booting, reducing the scrap rate, and so on. However, the early detection of hidden trouble of the printing press and early repair require fault information to be obtained in a timely by the operators or engineers. For those complex faults that the site operators cannot resolve, or the fault predictions can be obtained only by a professional data analysis, which would require recourse to off-site experts and engineers. But if this link can be achieved in the interaction on the Web, which will greatly, improve the efficiency and reduce costs [1]. At present, some well-known foreign brands already embedded networking modules in the printing press printing equipment, to provide better remote diagnostics service. At the same time, business is also growing demands for equipment network management, requiring easy access to relevant information anytime and anywhere. This article in response to this reality, designing the remote fault monitoring system based on the development of WAMP environment, collecting field data from TCP/IP, Web monitoring interface, implementing the browser remote login of users or experts, real-time monitoring and faults analysis.

\author{
Li Deng, Aiping Wang \\ School of Mechatronical Engineering and Automation \\ Shanghai University \\ Shanghai, China
}

\section{WAMP AND THE REMOTE MONITORING}

The open source software of Apache, MySQL and PHP (AMP) are commonly used to create and publish dynamic website or build server. The Apache HTTP Server (Apache) is an open webpage server of the Apache software foundation, and can be used in UNIX, Linux, Windows etc. the most computer operating system, and is one of the most popular Web application server. PHP is a server script language, which can make the site change to a dynamic web site and can be used as a substitute for Microsoft ASP.NET language, so it is widely used in the companies and individuals of all over the world. MySQL is a relational database management system, supporting the collaborative work of Apache and PHP. The advantages applied to Internet are as follow: small volume; high speed, high safety; low cost and other open source database server being relatively easier to manage. Typically, the LAMP set up under the Linux system has better performances in the web constructing and more popular; but the development of the WAMP under the Windows system has some advantages because of the friendly interface, plentiful software, simple operation [2].

The remote monitoring is a technique, developing by promoting of the continuous development of the computer and communication technology and the growing trend of network [3]. As the technology and production progress, people's demands for production and quality of products, security alerts and others have been steadily growing, and the remote monitoring draws more and more attention from all walks, and widely applied. In terms of industrial controls, the domestic many configuration software are integrated step by step based on the network connection and Web publishing, in order to meets the continued to expand demands in terms of network communication and control in industrial production [4].

\section{System Frame Of The Mote Fault Monitoring Of The PRINTING MACHINE}

The remote fault monitoring system of the printing press is designed on the base of the b/s and c/s mixed structure [5], as shown in Figure 1. The system is a three-tier structure, including the on-site equipment side, monitoring side of local and remote access side. The system consists of two subsystems: the data acquisition and storage communication subsystem of the c/s framework equipment, and the remote fault monitoring subsystem of the b/s framework. 


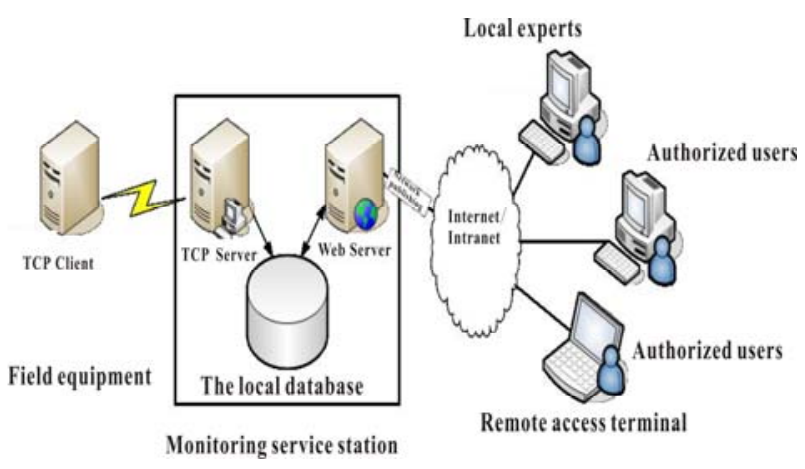

Figure 1. Topology structure of the remote fault monitoring system

(1) The on-site device side, namely, the printing machine and computer in printing workshop, Printing and IPC. The Gauss wheel turning with a standard Ethernet interface, the computer data acquisition is via Ethernet to the printing machine, used for field control, at the same time, as the realtime data transmission from the TCP client to the TCP server module station of the remote monitoring service.

(2) The monitoring station, including three main components of the TCP, remote Web servers and databases, covering the functions of the data received in real time, fault monitoring network dissemination and classifications of data access. To achieve the remote service interaction between the browser and the server, we use the dynamic Web page technology and the Hypertext pre-processing languages (PHP) interacting with the MySQL database to build a dynamic Web site, namely the server-side of the b/s structure, enabling the functions of users logging in to the management, monitoring the operation situations and fault data on-site printing press, and the remote diagnosis.

(3) The remote access end, the remote browser end of the $\mathrm{b} / \mathrm{s}$ structure. Users who are granted permission, experts and engineers, using a standard browser, access to the Internet/intranet, login into the remote fault monitoring platforms, to view and get fault data, condition monitoring and analysis.

\section{FIELD DATA ACQUisition AND StORAGE}

For the monitoring system, the acquisition and storage of the monitoring data and information are the basic and very key part of the system. This data-path is equivalent to the grass-roots of the system "transportation hub", which is divided into two layers of communication interfaces: one is the Ethernet communication interface between the industrial control computer and printer, and the other is the TCP/IP communication interface of the field data transmission between the industrial control computer and the remote monitoring server TCP/IP. Fig. 2 shows the basic flow of the design diagrams of the data real-time transmission on site.

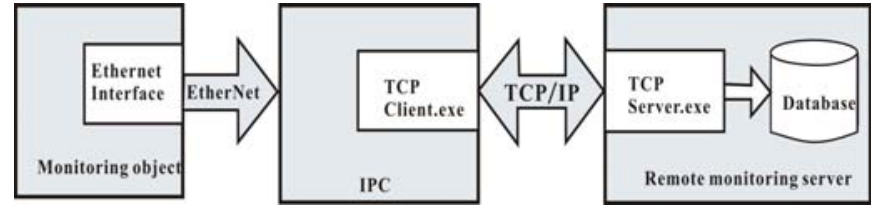

Figure 2. Data transmission diagram

The IPC at the production site and the device receive and monitor the data and information in real time via the Ethernet communication, directly to configuration, using to the control systems for production site. In the field, data use only for the operator to view the device status, and to make adjustments to the various components, and are not saved locally on the IPC. The remote monitoring server of the management requires not only the device current data also requires large amounts of historical data for Web publishing, for remote operation analysis and fault diagnosis of devices. This paper uses data transmission based on TCP protocol, the IPC setting the client side to request a connection and timing to send packets to the server.

As shown in Fig. 3, it shows the flow diagram for asynchronous communication between the servers and clients based on TCP protocol, using a streaming Socket (socket) technology to achieve the program monitoring to the network [3]. Server startup initialization includes: establishing socket, binding the network address, setting listen listening (such as port number, request queue length), then waiting for a client to connect. You can now create a socket client and request to connect to the server. After a successful connection, the exchanging of the data is carried out by means of a data flow between client and Server. When data transfer is completed, this session can be closed by closing the socket. Both client and server programs are wrote using VS2010 c \#, which can be achieved by the two classifications of the TcpListener in the NET Framework and TcpClient, and joining the Session mechanism to record session information.

Through the above methods, the real-time data of the printing presses on-site can be transferred from the IPC as the client to the server. Of course, in order to facilitate the classifications management of data, when sending and receiving packages, requiring being in a certain format and resolution. Every time the server successfully received data at a time, whose resolution you want to deposit types is derived from the data in the database tables. The main methods connecting MySQL are installing the driver and calling to a dynamic-link library or accessing through ODBC. Using the first method in this article, firstly, downloading NET driver program of the publishing (MySQL Connector Net.msi) and install it, then quoting the component of the MySQL.Data.dll in the engineering, and the Db connection MySQL can be called via using the encapsulation database access components, and basic database queries to the MySQL database connection is established and data manipulation. 


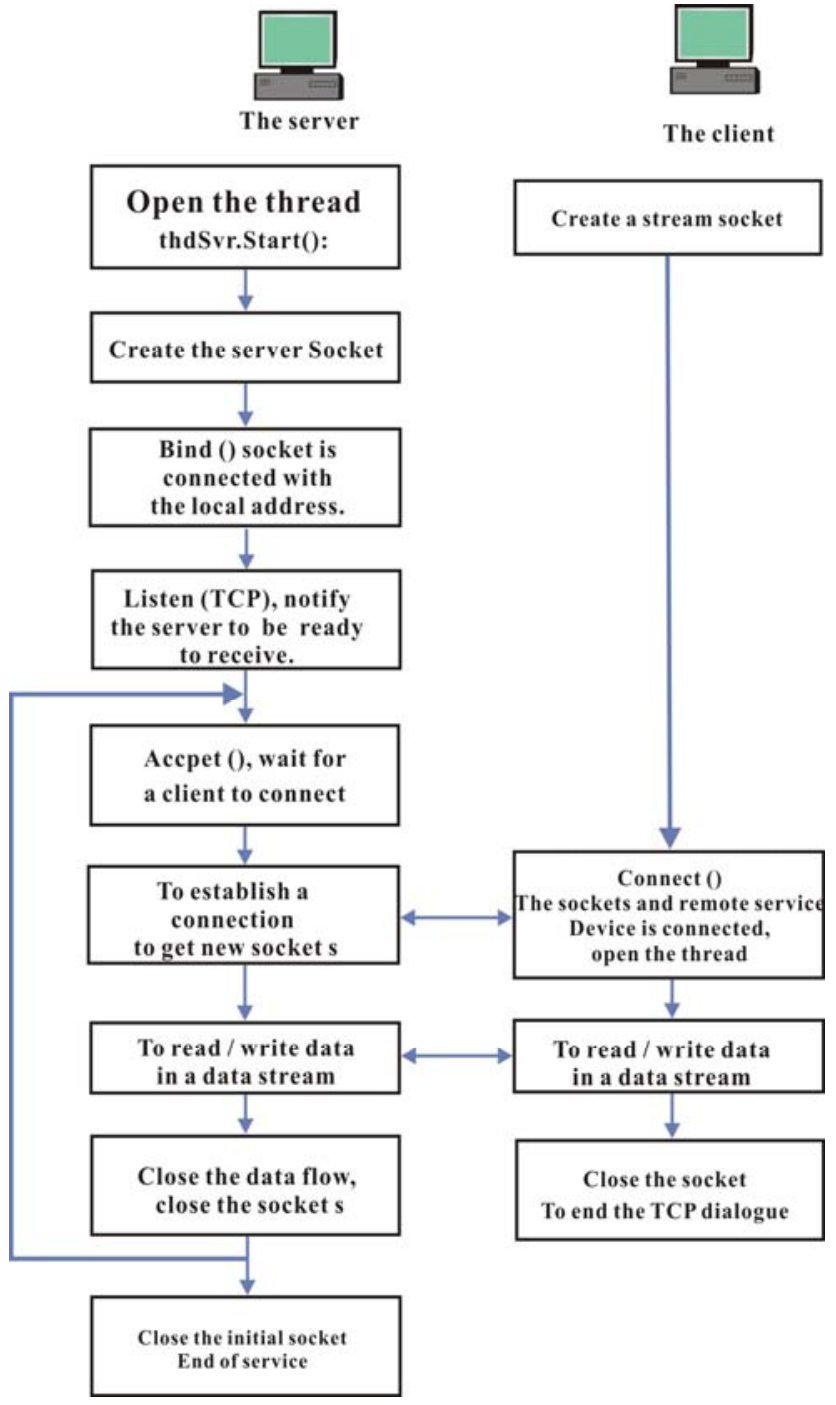

Figure 3. Schematic diagram of the client/server communication processes based on the TCP protocol

\section{Design of The Fault Monitoring Platforms}

The design of the remote fault monitoring platform is using the way of building website under the WAMP environment, its advantage is as follows: (1) using B/S mode to build network system, the remote access can be achieved using a standard browser, easy enterprise for management; (2) Apache, and PHP, and MySQL are open source, which can free using, its performance can meet SMEs needs; (3) the resources rich, easy reuse and II times development, late can achieved features extended.

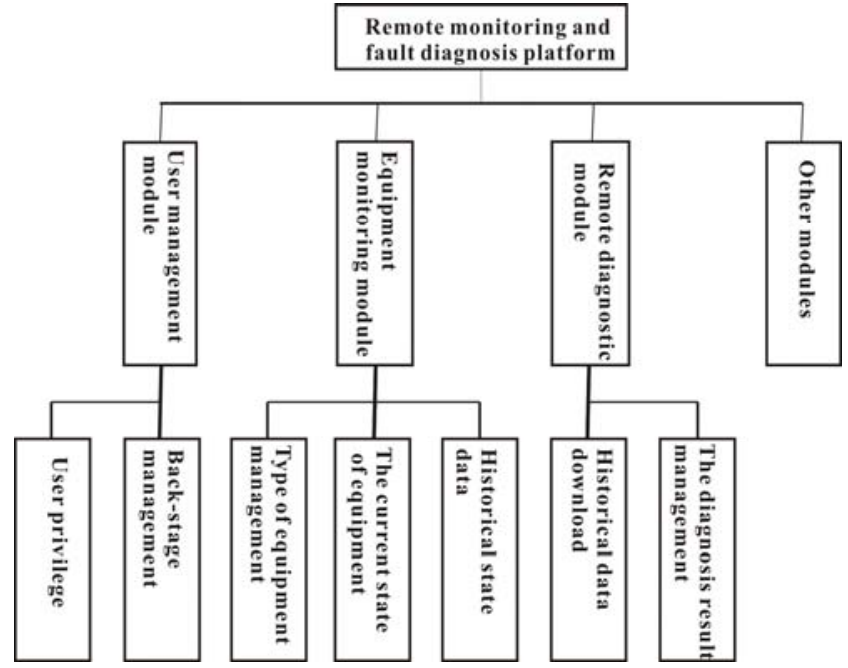

Figure 4. Remote fault monitoring platforms block diagram

As shown in Fig. 4, it is the modular design block diagram of the monitor platform. The remote fault monitoring platforms currently can be divided into three parts: the user management module, monitoring module of equipments and remote diagnostics modules. Other modules can be extended according to demand in the future.

(1)The user management module

System users, by permission, from high to low are administrators, diagnostic specialists and general monitors. The administrators are responsible for managing device information, load, permissions settings, and so on; the diagnostic specialist can remotely view and download device data for fault analysis and diagnosis, the general monitoring can only see current data and historical data on the page.

(2) The equipment Monitoring Module

The module is mainly used to display device state stored in the database, according to restrict user actions, such as the administrator can modify, add or remove device information, and other privileged users can only view the information.

(3)The Remote diagnostic module

The module is mainly manipulated by the diagnostic specialists. After the authorized specialist remote login system, they need to export historical data in a database for subsequent analysis.

\section{CONCLUSION}

This article is focused on the printing press to build a remote fault monitoring based on b/s structure of the WAMP environment systems, using the C/S system based on TCP protocol to achieve the data transferring between the IPC and the server platform. By Simulating tests, this paper put forward that the TCP client and server can transfer data from the press to the database; and the user login, status display and other functions could be realized via the remote monitoring system to interact with the database. The system is current; the database structure could be changed depending on the objects, which applies to similar situations. Next step can be further expanded for the remote diagnosis, and 
extending a study on the fault diagnosis algorithm, selecting the appropriate diagnosis of AI algorithms to analyze the historical data, establishing a remote fault diagnosis database and further integrating systematically with this article.

\section{REFERENCES}

[1] Jianmin Zhang, Xiaoli Xu, Baojie Xu, hongjun Wang. Contemporary Techniques of Condition Monitoring and Fault Diagnosis for Mechano-Electrical Systems [J], Journal of Beijing Institute of Technology,2004,24 (9): 751-756.

[2] Timothy Boronczyk, Elizabeth Naramore. Get started developing classic: PHP6, Apache and MySQL.[M]. Beijing Tsinghua University Press, 2009.

[3] Chengbo Yu, Jie Liu, Hongyan Tao. Research on Remote Monitor Technology of Equipment Based on Internet [J]. information and control, 2002,31 (3): 236-240
[4] Minglei Guo. Research of Web-based Remote Monitor and Control System [D]. Shenyang Ligong University, 2009.

[5] Zhengjia He, JinChen, Taiyong Wang. Mechanical fault diagnosis theory and application [m]. higher education in Beijing Publishing House, 2010.

[6] Yungang Zhang, Changchun Liu, Wei Liu, Fuzhi He. Remote Monitoring and Control System Based on Socket and Multithread [J]. Control Engineering of China, 2006,13 (2): 175-177

[7] Zhao Fagang, Chen Jin, Dong Guangming, Guo Lei. SOA-based remote condition monitoring and fault diagnosis system[J].International Journal of Advanced Manufacturing Technology,2010,46:1191-1200.

[8] Zeng Xiaohui, Li Manhua, Luo Wenlang. Research on a Remote Network Monitoring Model for Large-Scale Materials Manufacturing[C].International Conference on Mechanical Materials and Manufacturing Engineering, Nanchang,2011. 